\section{Toxicidade aguda da amônia em pacu (Piaractus mesopotamicus)}

\author{
Acutte toxicity of \\ ammonia in pacu \\ (Piaractus mesopotamicus)
}

Lucas Siqueira Manrique Pinheiro (1) Larissa Selini Dorce (10* Henrique Momo Ziemniczak (1) Claucia Aparecida Honorato da Silva (1) Dacley Hertes $\mathrm{Neu}$ (1)
Universidade Federal da Grande Dourados (UFGD), Dourados, MS, Brasil

* Correspondência: larissadorce@hotmail.com

Submetido: 9 fev 2021 | Aprovado: 24 mai 2021

DOI: http://dx.doi.org/10.7213/acad.2021.19004

Rev. Acad. Ciênc. Anim. 2021;19:e19004

\section{Resumo}

O Piaractus mesopotamicus é um peixe endêmico da bacia dos rios Paraná-Paraguai, apresentado boa adaptação em sistema intensivo de produção. Nesses sistemas, a amônia pode alcançar níveis extremos quando atividades nutricionais e de manejos não são corretas. Neste sentido, o objetivo do presente estudo foi avaliar a capacidade de tolerância de $P$. mesopotamicus expostos a diferentes concentrações de amônia e seu efeito na glicemia e concentração de amonia plasmática. Foram utilizados 36 exemplares com peso médio de $268 \pm 71 \mathrm{~g}$ e 20,7 $\pm 3,08 \mathrm{~cm}$ de comprimento padrão, dividios em 12 aquários contendo seis tratamentos e três exemplares por unidade experimental. Os tratamentos foram compostos por concentrações diferentes de $\mathrm{NH}_{4} \mathrm{Cl}$ nas seguintes doses: 0,0 (controle); 0,1;0,2;0,4;0,6; e 0,8 mg. L $^{-1}$ durante um período de 96 horas. Análises dos parâmetros de qualidade da água foram realizadas a cada 24 horas, a mortalidade contabilizada com intervalos de no máximo 12 horas e as análises sanguíneas foram realizadas nos animais sobreviventes ao teste. A amônia é tóxica para os pacus a partir de 1,674 mg. $\mathrm{L}^{-1}$ de $\mathrm{N}-\mathrm{NH}_{3}$, dose em que ocorreu a mortalidade de $50 \%$ da população em 66,75 horas e em $100 \%$ da população em 92,5 horas. $O$ elevado nível de glicose observado no tratamento 0,4 de $\mathrm{NH}_{4} \mathrm{Cl}$ pode ser atribuído ao estresse a que esses animais estavam acometidos, pois todos os animais estavam em um mesmo tempo de jejum. Conclui-se que $50 \%$ da população de $P$. mesopotamicus é tolerante até a 1,674 mg.L-1 de N-NH3 num período de 66,75 horas.

Palavras-chave: Aquicultura. $\mathrm{NH}_{3}$. Concentração letal.

\begin{abstract}
Piaractus mesopotamicus is an endemic fish from the Paraná-Paraguay river basin, well adapted to an intensive production system. In these systems, ammonia can reach extreme levels when nutritional and handling activities are not correct. In this sense, the aim of the present study was to evaluate the tolerance capacity of P. mesopotamicus exposed to different concentrations of ammonia and its effect on blood glucose and plasma ammonia concentration. Thirty-six specimens with an average weight of $268 \pm 71 \mathrm{~g}$ and $20.7 \pm 3.08 \mathrm{~cm}$ of
\end{abstract}


standard length were used, divided into 12 aquariums containing six treatments and three specimens per experimental unit. The treatments were composed of different concentrations of $\mathrm{NH}_{4} \mathrm{Cl}$ in the following doses: 0.0 (control), $0.1,0.2,0.4,0.6$, and $0.8 \mathrm{mg} . \mathrm{L}^{-1}$ over a 96 hour period. Analyzes of water quality parameters were performed every 24 hours, mortality at intervals of no more than 12 hours, and blood analyzes were performed on the animals surviving the test. Ammonia is toxic to pacus from $1.674 \mathrm{mg} \cdot \mathrm{L}^{-1}$ of $\mathrm{N}-\mathrm{NH}_{3^{\prime}}$ a dose in which the mortality of $50 \%$ of the population occurred in 66.75 hours, and $100 \%$ of the population at 92.5 hours. The high glucose level observed in the $0.4 \mathrm{NH}_{4} \mathrm{Cl}$ treatment can be attributed to the stress to which these animals were affected, since all animals were in the same fasting time. It is concluded that $50 \%$ of the P. mesopotamicus population is tolerant up to $1.674 \mathrm{mg} \cdot \mathrm{L}^{-1}$ of $\mathrm{N}-\mathrm{NH} 3$ in a period of 66.75 hours.

Keywords: Aquaculture. $\mathrm{NH}_{3}$. Lethal concentration.

\section{Introdução}

A aquicultura é uma das atividades com maior crescimento em nível mundial, obtendo taxa em torno de 6,5\% ao ano (Leung e Bates, 2013). Em 2018 a produção de pescados mundial resultou em 178,5 milhões de toneladas, sendo 82,1 milhões de toneladas provenientes da aquicultura, o que representa US\$263,6 bilhões (FAO, 2020). A intensificação da produção sem a perda de desempenho das espécies é um dos desafios que a aquicultura enfrenta (Abdel-Tawwab et al., 2008). Atrelado a isso, está a constante pressão mundial no fornecimento de alimentos com preço acessível e praticados com sustentabilidade.

Dentre as espécies de peixes nativas, o pacu (Piaractus mesopotamicus) tem boa aceitação comercial, com enorme importância para pesca comercial, ocorrendo a criação em sua maioria nas regiões Sudeste e Centro-Oeste do Brasil (Serafini, 2010). É uma espécie que levanta interesse para piscicultura pelo grande valor comercial, facilidade na obtenção de larvas e adaptação à alimentação artificial (Urbinati et al., 2010), ganhando destaque também no cultivo em tanques-rede devido a sua fácil adaptação ao sistema de cultivo e pela baixa exigência nutricional; exemplo é a exigência proteica, inferior a outras espécies (Fernandes et al., 2000; Signor et al., 2010; Khan et al., 2020).

Mesmo que a espécie apresente essa característica nutricional, as dietas fornecidas devem ser balanceadas e completas, no intuito de prover nutrientes disponíveis ao desempenho e atividades fisiológicas, bem como evitar perdas desses elementos para o meio, o que contribui para a redução da qualidade da água e diretamente incrementa a quantidade de amônia na água. Por estarem relacionados à fração proteica da dieta e seu processo cataólico, esses elementos geram resíduos nitrogenados que são eliminados nas fezes ou em forma de amônia pelas brânquias (Boyd, 1992). O acúmulo de amônia na água pode resultar em aumento nas concentrações desse parâmetro no sangue e tecidos de peixes, o que pode ocasionar a redução do desempenho zootécnico dos animais, e concentrações mais elevadas podem provocar toxicidades subletal e letal (Dinesh, 2013).

Os testes de toxicidade são aplicados como procedimento necessário para identificar ou mensurar a presença de uma ou mais substâncias causadora de estresse biológico no organismo, sendo essas substâncias isoladas ou conciliadas aos fatores ambientais (Long, 1982; Barbieri, 2007). O conhecimento desses valores tem implicação direta na aquicultura, pois é possível determinar em quais condições e em quanto tempo os prejuízos relacionados a esta variável se tornarão efetivos, podendo, assim, tomar-se medidas de manejo corretivo e evitar a perda de animais.

O objetivo deste trabalho foi avaliar a capacidade de tolerância de $P$. mesopotamicus expostos a diferentes concentrações de amônia e seu efeito na glicose e concentração de amônia plasmática.

\section{Material e métodos}

O ensaio in vivo, aprovado pela Comissão de Ética no Uso de Animais (CEUA) da Universidade Federal da Grande Dourados (UFGD), sob parecer $n^{\circ} 39 / 2016$, foi realizado com juvenis de pacu (268 $\pm 71 \mathrm{~g}$ ), aclimatados em caixa de $500 \mathrm{~L}$ provida de aeração e com uma renovação diária de $70 \%$ do seu volume durante cinco dias. Posteriormente, os peixes foram divididos em 12 unidades experimentais de 60 
$L$ de água contendo três peixes, em um total de seis peixes por tratamento, em sistema de recirculação, com fotoperíodo de 12 horas de luz. Os parametros da água foram monitorados uma vez ao dia durante o ensaio experimental. Utilizou-se o equipamento EcoSense DO200A (temperatura e oxigênio dissolvido), Digital Phmetro Lcd Pen Type Ph Meter $(\mathrm{pH})$ e condutivímetro digital (condutividade elétrica).

A avaliação da toxicidade da amônia para pacu foi realizada segundo o método descrito por Meyer et al. (1982). Foram utilizados cinco concentrações de cloreto de amônia durante 96 horas: 0,1; 0,2; 0,$4 ; 0,6$; e 0,8 mg. L-1. Um grupo controle também foi preparado nas mesmas condições dos tratamentos, mas sem a presença da substância-teste, sendo adicionada água destilada em substituição ao cloreto de amônia. Os peixes permaneceram em sistema estático com aeração constante. A sobrevivência foi verificada a cada hora nas primeiras 8 horas e, posteriormente, verificada com intervalo de 12 horas.

Para atingir as concentrações de $\mathrm{N}-\mathrm{NH}_{3}$ desejadas, utilizou-se o cloreto de amônia $\left(\mathrm{NH}_{4} \mathrm{Cl}\right)$ como veículo (Tabela 1). As amostras para análise de amônia não iozinada foram coletadas 30 minutos após a adição do cloreto de amônia nos aquários, e refrigeradas a $-18{ }^{\circ} \mathrm{C}$ para posterior análise em espectrofotômetro. As análises de amônia foram realizadas utilizando o protocolo do teste colorimétrico da Alfakit, de amônia indotest. Posteriormente, para completar o teste, analisou-se em espectrofotômetro Spectrumlab $22 \mathrm{pc}$ a absorbância das amostras em comprimento de onda $630 \mathrm{~nm}$.

Tabela 1 - Concentrações (mg. $\left.\mathrm{L}^{-1}\right)$ nominais de cloreto de amônia $\left(\mathrm{NH}_{4} \mathrm{Cl}\right)$ e amônia $\left(\mathrm{N}-\mathrm{NH}_{3}\right)$ observadas na água dos aquários

\begin{tabular}{lllllll}
\hline & \multicolumn{6}{c}{ Concentração observada (mg.t.-1) } \\
\cline { 2 - 7 } $\mathbf{N H}_{4} \mathrm{Cl}$ & 0,00 & 0,10 & 0,20 & 0,40 & 0,60 & 0,80 \\
$\mathbf{N}^{-} \mathrm{NH}_{3}$ & 0,20 & 0,39 & 0,94 & 1,49 & 1,67 & 1,60 \\
\hline
\end{tabular}

As concentrações foram definidas com base nos valores observados na literatura para espécies neotropicais: $\mathrm{C}_{\mathrm{L} 50}$ de $\mathrm{NH}_{3}$ em 24 horas variando de 0,66 a 0,85 mg.L-1 (Person-Le Ruyet et al., 1995) e 0,82 mg.L-1 com valor médio de $\mathrm{CL}_{50}$ em 96 horas de $\mathrm{NH}_{3}$ para peixes em geral (Gentzkow e Masen, 1942).
Ao término do experimento (96 horas) foram coletadas amostras de sangue por punção da veia caudal dos peixes sobreviventes. A glicose foi mensurada utilizando o medidor G-Tech Free, que tem a capacidade de leitura variando de 10 a $600 \mathrm{mg}^{-\mathrm{dL}^{-1}}$ de glicose, e a amônia foi determinada pelo método de Nesslerização (Erdogan et al., 2005). Um volume adequado de extrato ácido era transferido para um tubo com água destilada em um volume final de 2,0 $\mathrm{ml}$ e adicionado $0,5 \mathrm{ml}$ de reativo de Nessler. A leitura óptica foi realizada em espectrofotômetro em $420 \mathrm{~nm}$. As concentrações de amônia foram estimadas contra um padrão de amônia contendo 100 nmols de $\mathrm{NH}_{4} \mathrm{Cl}$.

Para a verificação das concentrações letais $\left(\mathrm{CL}_{50}\right.$ e $\left.\mathrm{CL}_{100}\right)$, dos tempos letais $\left(\mathrm{TL}_{50}\right.$ e $\left.T \mathrm{~L}_{100}\right)$ de amônia e da sobrevivência dos peixes, utilizou-se estatística descritiva. Foram anotados os tempos decorridos da morte dos animais em cada repetição e calculados os tempos letais $\left(T_{50}\right)$ e $\left(T L_{100}\right)$ com auxílio do pacote Excel do Microsoft Office.

Este ensaio foi analisado em delineamento experimental inteiramente casualizado consistindo de seis tratamentos com seis repetições, sendo os peixes considerados como repetição. Os dados dos parâmetros hematológicos (glicose e amônia) obedeceram à distribuição normal e foram submetidos ao teste de ANOVA em $5 \%$ de probabilidade. Quando observadas diferenças estatísticas, aplicou-se o teste de Tukey. O mesmo foi utilizado para as análises de qualidade de água e análise de correlação de Pearson, para verificar os possíveis efeitos entre as variáveis e os dias de exposição. O programa utilizado foi o Statistic 7.1 (Statsoft, 2005).

\section{Resultados e discussão}

Logo após a adição do $\mathrm{NH}_{4} \mathrm{Cl}$, ocorreram comportamentos atípicos nos peixes dos tratamentos com inclusão de 0,4; 0,6 e 0,8 mg. $\mathrm{L}^{-1}$ de $\mathrm{NH}_{4} \mathrm{Cl}$. Os animais começaram a se debater e mostraram-se agitados. Apresentaram, ainda, batimento opercular acelerado, maior movimentação e natação errática. Esses comportamentos são característicos de animais que estão sob efeitos subletais da amônia (Damato e Barbieri, 2011). Abreu et al. (2012) relatam que o pacu $(279.8 \pm 48.9 \mathrm{~g})$ é um peixe resistente à intoxicação por amônia, sendo expostos a concentrações elevadas $\left(0,0078\right.$ e $\left.0,078 \mathrm{NH}_{4} \mathrm{Cl} \mathrm{g} / \mathrm{L}\right)$ por 24 horas. Entretanto 
há poucos estudos comportamentais sobre os efeitos da amônia em peixes nativos.

Ocorreram mortalidades apenas nos tratamentos 0,6 e $0,8 \mathrm{mg} \cdot \mathrm{LL}^{-1}$ de $\mathrm{NH}_{4} \mathrm{Cl}$ (Figura 1), apesar de a literatura reportar valores superiores na concentração que leva à mortalidade de pacu: 6,68 mg.L-1 de N-NH (Martinez et al., 2006), 3,00 mg.L-1 de N-NH

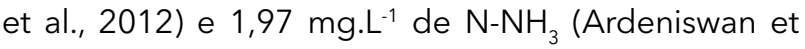
al., 2017). Ressalta-se, no entanto, que alguns fatores devem ser considerados sobre as concentrações tóxicas de $\mathrm{N}-\mathrm{NH}_{3}$, tais como tamanho da espécie, temperatura e qualidade de água, principalmente o pH (Martinez et al., 2006), que embora no atual estudo não tenha sido verificada alteração brusca, ficou acima de 7,0, propenso a maior toxicidade por $\mathrm{NH}_{3}$ (devido à característica básica). Além disso, mesmo o oxigênio sendo constante nos aquários, a temperatura da água permaneceu em torno de $23^{\circ} \mathrm{C}$, o que contribui para a não conversão da amônia em nitrato (Henze et al., 2002). A influência do $\mathrm{pH}$ em relação à toxicidade de $\mathrm{NH}_{3}$ foi reportada para Cyprinus carpio, que obtiveram $60 \%$ de mortalidade com pH 8 e 1,85 mg.L-1 de $\mathrm{NH}_{3}$ e $100 \%$ com pH 9 e 2,16 mg.L-1 de $\mathrm{NH}_{3}$ em 96 horas (Schmidt-Nielsen, 1997); portanto, valores superiores ao obtido no atual estudo. $\mathrm{O}$ pH não foi influenciado pela adição de $\mathrm{NH}_{4} \mathrm{Cl}$, porém, a partir de 16 horas, ocorreu mortalidade com a concentração de $0,8 \mathrm{mg}$.'${ }^{1}$ de $\mathrm{NH}_{4} \mathrm{Cl}$ (Tabela 2)

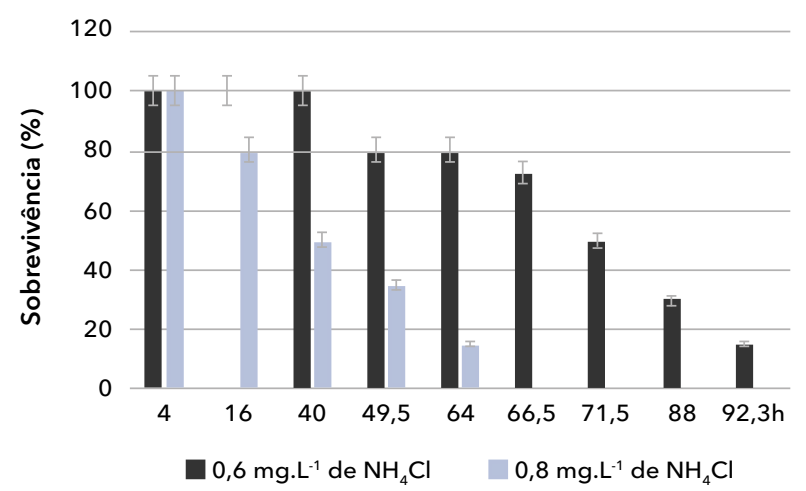

Figura 1 - Sobrevivência de pacu ao longo do tempo expostos a concentração de 0,6 e $0,8 \mathrm{mg} \cdot \mathrm{L}^{-1}$ de $\mathrm{NH}_{4} \mathrm{Cl}$.

Barbieri e Bondioli (2015), verificaram $\mathrm{CL}_{50}$ de $1,97 \mathrm{mg} \cdot \mathrm{L}^{-1}$ de $\mathrm{N}-\mathrm{NH}_{3}$ em 96 horas para juvenis de pacu com 1,2 gramas de peso corporal; no presente estudo, verificou-se $\mathrm{CL}_{50}$ de $1,67 \mathrm{mg} \cdot \mathrm{L}^{-1} \mathrm{em} 66$ horas.
O menor valor de toxidade da amônia para os peixes do atual experimento podem estar relacionado ao tamanho dos peixes, em função da superfície corporal para absorção da amônia e da maior taxa respiratória em indivíduos jovens (Martinez et al., 2006).

A temperatura, $\mathrm{pH}$ e oxigênio dissolvido permaneceram dentro dos níveis considerados confortáveis para o cultivo de peixes (Boyd, 1992) e estão apresentados na Tabela 2. A toxicidade da amônia é responsiva à temperatura da água (Ardeniswan et al., 2017), mas a oscilação de 22 e $26{ }^{\circ} \mathrm{C}$ obervada no presente estudo não foi o motivo da intoxicação e está provavelmente relacionada à inclusão do cloreto de amônia na água.

A água dos aquários que receberam os níveis de $0,6 \mathrm{mg} \cdot \mathrm{L}^{-1}$ de $\mathrm{NH}_{4} \mathrm{Cl}$ apresentou queda nas concentrações de oxigênio dissolvido nas primeiras 24 horas de experimento, retornando aos níveis normais posteriormente. Os valores de $\mathrm{pH}$ oscilaram ao longo do período experimental ( $p>0,05$ ), independentemente do tratamento. Todos os valores de $\mathrm{pH}$ registrados no final das 96 horas de experimento foram maiores do que os valores das primeiras horas de experimento, oscilando entre 7,0 e 7,8, o que pode ser atribuído à falta de renovação da água.

Houve diferença significativa $(p<0,05)$ para a condutividade elétrica da água ao longo do experimento, como pode ser verificado na Tabela 2. Esse aumento constante na condutividade elétrica está relacionado a dois pontos principais: inclusão de cloreto de amônio na água e perda de íons dos animais, interferindo na osmorregulação, como relatado por Peyghan e Takami (2002). Uma vez que os animais do tratamento com adição de $0,8 \mathrm{mg} \cdot \mathrm{L}^{-1}$ de $\mathrm{NH}_{4} \mathrm{Cl}$ vieram a óbito, esses valores foram semelhantes aos da água dos animais que foram submetidos à água sem a inclusão do sal.

Houve correlação $(p<0,05)$ negativa $(-0,43)$ da temperatura da água com o oxigênio dissolvido e com o pH da água dos aquários $(-0,31)$ (Tabela 3). Quando houve aumento de temperatura, o oxigênio foi consumido com maior rapidez pelos peixes, o que é comum em peixes teleósteos (Abimorad et al., 2009), bem como redução no $\mathrm{pH}$ da água, que possivelmente se deu por influência do gás carbônico expelido pelos animais durante o processo respiratório (Thomas, 1990), que estava aumentado nos peixes com maior teor de cloreto de amônio e visualizado por meio dos batimentos operculares acelerados. 
Tabela 2 - Parâmetros da qualidade de água durante o período de experimental de pacu expostos a concentrações de $\mathrm{NH}_{4} \mathrm{Cl}\left(\mathrm{mg} \cdot \mathrm{L}^{-1}\right)$

\begin{tabular}{|c|c|c|c|c|c|c|c|}
\hline & \multicolumn{6}{|c|}{ Concentração de $\mathrm{NH} 4 \mathrm{Cl}\left(\mathrm{mg} \cdot \mathrm{L}^{-1}\right)$} & \multirow{2}{*}{ Valor $\mathrm{p}$} \\
\hline & 0,0 & 0,1 & 0,2 & 0,4 & 0,6 & 0,8 & \\
\hline Temperatura $\left({ }^{\circ} \mathrm{C}\right)$ & 23,83 & 23,20 & 23,11 & 23,00 & 23,50 & 23,10 & 0,27 \\
\hline Oxigênio dissolvido (mg. $\left.\mathrm{L}^{-1}\right)$ & 4,59 & 5,74 & 4,49 & 5,35 & 5,11 & 5,66 & 0,11 \\
\hline $\mathrm{pH}$ & 7,58 & 7,53 & 7,36 & 7,37 & 7,28 & 7,61 & 0,05 \\
\hline Condutividade elétrica $\left(\mu \mathrm{S} . \mathrm{cm}_{-1}\right)$ & $110,40^{e}$ & $612,50^{d}$ & $1.125,40^{c}$ & $1.748,00^{\mathrm{b}}$ & $2.305,00^{\mathrm{a}}$ & $110,40^{e}$ & $<0,001$ \\
\hline
\end{tabular}

Nota: Letras diferentes na mesma linha diferem entre si ao nível de 5\% de significância utilizando o teste Tukey.

Houve correlação positiva $(0,65)$ entre $\circ \mathrm{pH}$ da água e os dias de experimentação (Tabela 3), com aumento dos valores de $\mathrm{pH}$ da água. Essa correlação moderada pode explicar a $\mathrm{CL}_{50}$ e a $\mathrm{CL}_{100}$ dos tratamentos, pois o aumento desse parâmetro pode ter contribuído de modo a favorecer uma potencialidade maior na toxicidade da amônia, e os peixes dos tratamentos 0,6 e $0,8 \mathrm{mg} \cdot \mathrm{L}^{-1}$ de $\mathrm{NH}_{4} \mathrm{Cl}$ não suportaram, vindo a óbito.

A condutividade elétrica permaneceu estável ao longo de todo o período de experimento, entretanto, foi distinta entre as inclusões de cloreto de amônia na água $(\mathrm{p}<0,05)$. Por ser um sal, o $\mathrm{NH}_{4} \mathrm{Cl}$ tem capacidade de alterar a condutividade elétrica da água. Houve correlação positiva moderada entre a condutividade elétrica e o $\mathrm{pH}$ da água dos aquários, porém, sem causar danos aos animais.

Observou-se aumento dos níveis de glicose plasmática conforme o aumento de concentração de $\mathrm{NH}_{4} \mathrm{Cl}$ e redução dos níveis de amônia no sangue (Tabela 4). Os peixes submetidos à concentração de $0,4 \mathrm{mg} \cdot \mathrm{L}^{-1}$ apresentaram aumento na atividade natatória pela presença da $\mathrm{NH}_{4} \mathrm{Cl}$ na água dos aquários, assim como maiores valores de glicose.

Tabela 3 - Correlação de Pearson* para os principais parâmetros da qualidade de água de pacus expostos a concentrações de $\mathrm{NH}_{4} \mathrm{Cl}$ (mg.L-1)

\begin{tabular}{lcccc}
\hline & Temperatura & Oxigênio & pH & Condutividade \\
\hline Temperatura & - & - & - & - \\
Oxigênio & $-0,43$ & - & - & - \\
pH & $-0,31$ & 0,61 & - & - \\
Condutividade $\left(\mu \mathrm{S}_{\text {.cm }}{ }^{-1}\right)$ & $-0,08$ & $-0,05$ & $-0,43$ & - \\
Dias de experimento & $-0,20$ & 0,23 & $0,65^{\star}$ & $-0,23$ \\
\hline
\end{tabular}

Nota: Dados tabulados e rodados no programa Statistic 7.1 (Statsoft, 2005).

Tabela 4 - Concentração de glicose e amônia $\left(m g \cdot \mathrm{dL}^{-1}\right)$ sanguíneas de pacu expostos a concentrações de $\mathrm{NH}_{4} \mathrm{Cl}$ $\left(\mathrm{mg} \cdot \mathrm{L}^{-1}\right)$

\begin{tabular}{|c|c|c|c|c|c|c|c|}
\hline & \multicolumn{6}{|c|}{ Tratatamento com NH4Cl (mg. $\left.\mathrm{L}^{-1}\right)$} & \multirow{2}{*}{ Valor $p$} \\
\hline & 0,0 & 0,1 & 0,2 & 0,4 & 0,6 & 0,8 & \\
\hline Glicose & $43,4^{c}$ & $52,8^{c}$ & $83,8^{b}$ & $120,2^{\mathrm{a}}$ & * & * & 0,01 \\
\hline
\end{tabular}

Nota: *Não havia peixes para coleta. Letras diferentes na mesma linha diferem entre si ao nível de $5 \%$ de significância utilizando o teste Tukey. 
É notória a relação de que quanto maior a quantidade de amônia na água, maior o valor de glicose no sangue. Esta hiperglicemia é uma resposta endócrina ao estresse, o que resulta na rápida mobilização de energias de reserva, podendo ser considerada um mecanismo adaptativo que permite ao corpo um aumento de energia demandada durante a exposição a estressores (Evans e Cameron, 1986; Wilkie, 1997; Das et al., 2004). Quanto maiores as concentrações de amônia na água, mais responivos são os níveis glicêmicos no sangue dos peixes (Person-Le et al., 1995; Barbieri e Bondioli, 2015; Ardeniswan et al., 2017). Esse fato também foi observado em Cirrhinus mrigala, que apresentou aumento progressivo de glicose com aumento da quantidade de amônia na água (Wilkie, 1997).

Para suportar esse período de estresse, os peixes acabam utilizando mecanismos e atitudes compensatórias, como elevar a glicose disponível no sangue, conforme observado nos resultados do atual estudo bem como por Abreu et al. (2012) com a mesma espécie. Talvez essa seja uma condição da espécie para poupar energia a fim de superar essa situação de estresse ou, ainda, uma estratégia adaptativa em esperar que as condições melhorem (Israeli-Weinstein e Kimmel, 1998). O estresse relacionado à intoxicação também pode explicar os resultados sanguíneos encontrados no presente trabalho. Venturini et al.,(2018) relatam que o estresse por exposição a agrotóxico aumenta o cortisol e glicose no sangue de matrinxãs (Brycon amazonicus), sendo esses parâmetros os princípais indicadores de estresse em peixes (Barton, 2000).

A brânquia é o principal local de excreção de amônia em peixes (Chen e Kou, 1993; Boyd e Tucker, 1998; Wilkie, 2002; Weihrauch et al., 2009), e com o aumento da sua concentração na água ocorre uma redução no gradiente de difusão entre o sangue e o meio externo, aumentando a quantidade de amônia no sangue e nos tecidos e gerando sérios problemas fisiológicos (Cavero et al., 2004) e estresse nos peixes.

Portanto, visando obter valores de referência para peixes, esses estudos de tolerância são extremamente importantes principalmente em situações de criação intensiva, onde há aumento da densidade de estocagem dos animais, aumento da oferta de alimento e consequente aumento na excreção, fazendo com que o meio tenha maiores quantidades de amônia. Nesse sentido, relações estreitas com as váriáveis de água devem ser estabelecidas, pois alguns manejos rotineiros podem ajudar o animal a se manter vivo durante períodos em que haja uma situação de estresse.

\section{Conclusão}

A amônia é tóxica para os pacus com peso aproximado de 270 gramas, a partir de 1,67 mg.L-1 de $\mathrm{N}-\mathrm{NH}_{3}$. Nesta concentração ocorre mortalidade de $50 \%$ da população em 66,75 horas e em $100 \%$ da população em 92,5 horas.

O tratamento com $1,49 \mathrm{mg} \cdot \mathrm{L}^{-1}$ de $\mathrm{N}-\mathrm{NH}_{3}\left(0,4 \mathrm{mg} \cdot \mathrm{L}^{-1}\right.$ de $\mathrm{NH}_{4} \mathrm{Cl}$ ) ao final das 96 horas de experimento, começou a demonstrar os primeiros sintomas de desconforto ocasionado pela concentração de amônia no ambiente, sintomas similares aos apresentados nos tratamentos que registraram mortalidades.

\section{Referências}

Abdel-Tawwab M, Abdel-Rahman AM, Ismael NEM. Evaluation of commercial live baker's yeast, Saccharomyces cerevisiae as a growth and immunity promoter for fry Nile tilapia Oreochromis niloticus challenged in situ with Aeromonas hydrophila. Aquaculture. 2008;280(1-4):185-9.

Abimorad EG, Favero GC, Castellani D, Garcia F, Carneiro DJ. Dietary supplementation of lysine and/or methionine on performance, nitrogen retention and excretion in pacu Piaractus mesopotamicus reared in cages. Aquaculture. 2009; 295(3-4):266-70.

Abreu JS, Esteves FR, Urbinati EC. Stress in pacu exposed to ammonia in water. Rev Bra Zootec. 2012;41(7):1555-60.

Ardeniswan, Dara F, Sukmawati F. Acute toxicity $\left(\mathrm{LC}_{50}\right)$ of ammonia to carp fish (Cyprinus carpio) at different $\mathrm{pH}$ Levels. IOP Conf Ser: Earth Environ Sci. 2017;60:012037.

Barbieri E, Bondioli ACV. Acute toxicity of ammonia in Pacu fish (Piaractus mesopotamicus, Holmberg, 1887) at different temperatures levels. Aquac Res. 2015;46(3):565-71.

Barbieri E. Use of oxygen consumption and ammonium excretion to evaluate the sublethal toxicity of cadmium and zinc on Litopenaeus schmitti (Burkenroad, 1936, Crustacea). Water Environ Res. 2007;79(6):641-6. 
Barton BA. Salmonid fishes differ in their cortisol and glucose responses to handling and transport stress. N Am J Aquac. 2000;62(1):12-8.

Boyd CE, Tucker CS. Pond aquaculture water quality management. Boston: Kluwer Academic Publishers; 1998. 268 p.

Boyd CE. Water quality management for pond fish culture. Amsterdam: Elsevier; 1992. 318 p.

Cavero BAS, Pereira-Filho M, Bordinhon AM, Fonseca FAL, Ituassú DR, Roubach R, et al. Tolerância de juvenis de pirarucu ao aumento da concentração de amônia em ambiente confinado. Pesq Agropec Bras. 2004;39(5):513-16.

Chen JC, Kou YZ. Accumulation of ammonia in the hemolymph of Penaeus monodon exposed to ambient ammonia. Aquaculture. 1993;109(2):177-85.

Damato M, Barbieri E. Determinação da toxicidade aguda de cloreto de amônia para uma espécie de peixe (Hyphessobrycon callistus) indicadora regional. Mundo Saude. 2011;35(4):401-7.

Das PC, Ayyappan S, Jena JK, Das BK. Acute toxicity of ammonia and its sub- lethal effects on selected haematological and enzymatic parameters of mrigal, Cirrhinus mrigala (Hamilton). Aquac Res. 2004;35(2):134-43.

Dinesh B, Ramesh M, Poopal RK. Effect of ammonia on the electrolyte status of an Indian major carp Catla catla. Aquac Res. 2013;44(11):1677-84.

Erdogan O, Hisar O, Köroglu G, Ciltas A. Sublethal ammonia and urea concentrations inhibit rainbow trout (Oncorhynchus mykiss) erythrocyte glucose-6-phosphate dehydrogenase. Comp Biochem Physiol C Toxicol Pharmacol. 2005;141(2):145-50.

Evans DH, Cameron JN. Gill ammonia transport. J Exp Zool. 1986;239(1):17-23.

FAO - Food and Agriculture Organization of the United Nations 2020. The state of world fisheries and aquaculture: sustainability in action. Rome: FAO; 2020. 206 p.

Fernandes JBK, Carneiro DJ, Sakomura NK. Fontes e níveis de proteína bruta em dietas para alevinos de pacu (Piaractus mesopotamicus). R Bras Zootec. 2000;29(3):646-53.
Gentzkow CJ, Masen JM. An accurate method for the determination of blood urea nitrogen by direct nesslerization. J Biol Chem. 1942;143(2):531-44.

Henze M, Harremoes P, Arvin E, La Cour Jansen J. Wastewater treatment: Biological and chemical processes. 3 ed. Berlim: Springer; 2002. 430 p.

Israeli-Weinstein D, Kimmel E. Behavioral response of carp (Cyprinus carpio) to ammonia stress. Aquaculture. 1998;165(1-2):81-93.

Khan KU, Gous RM, Mansano CFM, Nascimento TMT, Romaneli RS, Rodrigues AT, et al. Response of juvenile pacu (Piaractus mesopotamicus Holmberg, 1887) to balanced digestible protein. Aquac Res. 2020;51(9);3729-40.

Leung TLF, Bates AE. More rapid and severe disease outbreaks for aquaculture at the tropics: implications for food security. $J$ Appl Ecol. 2013;50(1):215-22.

Long Er. An assessment of marine pollution in Puget Sound. Mar Pollut Bull. 1982;13(11):380-3.

Martinez CBR, Azevedo F, Winkaler EU. Toxicidade e efeitos da amônia em peixes neotropicais. In: Cyrino JEP, Urbinati EC, editores. Tópicos especiais em biologia aquática e aquicultura. Jaboticabal: Sociedade Brasileira de Aquicultura e Biologia Aquática; 2006. p. 81-95.

Meyer BN, Ferrigni NR, Putnam JE, Jacobsen LB, Nichols DE, McLaughlin JLM. Brine shrimp: a convenient general bioassay for active plant constituents. Planta Med. 1982;45(5):31-4.

Peyghan R, Takami GA. Histopathological, serum enzyme, cholesterol and urea changes in experimental acute toxicity of ammonia in commom carp Cyprinus carpio and use of natural zeolite for prevention. Aquac Int. 2002;10:317-25.

Person-Le Ruyet J, Chartois H, Quemener L. Comparative acute ammonia toxicity in marine fish and plasma ammonia response. Aquaculture. 1995;136(1-2):181-94.

Schmidt-Nielsen K. Animal Physiology: Adaptation and Environment. 5 ed. Cambridge, UK: Cambridge University Press; $1997.617 \mathrm{p}$. 
Serafini MA. Cruzamento dialélico interespecífico entre pacu Piaractus mesopotamicus e tambaqui Colossoma macropomum [tese]. Lavras: Universidade Federal de Lavras; 2010. 68 p.

Signor AA, Boscolo WR, Feiden A, Bittencourt F, Coldebella A, Reidel A. Proteína e energia na alimentação de pacus criados em tanques-rede. R Bras Zootec. 2010;39(11):2336-41.

Thomas P. Molecular and biochemical responses of fish to stressors and their potential use in environmental monitoring. Am Fisheries Soc Symp; 1990;8:9-28.

Urbinati EC, Gonçalves FD, Takahashi LS. Pacu (Piractus mesopotamicus). In: Baldisserotto B, Gomes LC (Org.). Espécies nativas para piscicultura no Brasil. 2 ed. Santa Maria: UFSM; 2010. p. 205-44.
Venturini FP, Moraes FD, Rossi PA, Avilez IM, Shiogiri NS, Moraes G. A multi-biomarker approach to lambda-cyhalothrin effects on the freshwater teleost matrinxa Brycon amazonicus: single-pulse exposure and recovery. Fish Physiol Biochem. 2018;45(1):341-53.

Weihrauch D, Wilkie MP, Walsh PJ. Ammonia and urea transporters in gills of fish and aquatic crustaceans. J Exp Biol. 2009;212(Pt 11):1716-30.

Wilkie MP. Ammonia excretionn and urea handling by fish gills: Present understanding and future research challenges. J Exp Zool. 2002;293(3);284-301.

Wilkie MP. Mechanism of ammonia excretion across fish gills. Comp Biochem Physiol A Physiol. 1997;118(1):39-50. 